\title{
ESTRATÉGIAS DE ENFRENTAMENTO NA REABILITAÇÃO DO TRAUMATISMO RAQUIMEDULAR
}

\author{
Maria Eloá Moreira da Silva Martins Pereira', \\ Tereza Cristina Cavalcanti Ferreira de Araujo²
}

\begin{abstract}
RESUMO - Centros de pesquisa internacionais vêm realizando estudos sobre traumatismo raquimedular, no intuito de subsidiar intervenções voltadas para reabilitação e qualidade de vida de portadores de lesão medular. Assim, considerando os atuais interesses científicos e profissionais da Psicologia da Saúde, a presente investigação teve por objetivo compreender as estratégias de enfrentamento adotadas pelo paciente e seu familiar/acompanhante durante a participação em um programa de reabilitação. A amostra foi constituída por seis díades paciente/familiar. Os participantes foram avaliados, antes do ingresso no programa e ao seu término, através da aplicação da Escala de Modos de Enfrentar Problemas e de entrevista semi-estruturada. Os dados obtidos revelaram mudanças nas modalidades estratégicas empregadas entre a etapa de pré-reabilitação (religiosidade, pensamento positivo e busca de suporte social) e a etapa de pós-reabilitação (focalização no problema, pensamento positivo e busca de suporte social).
\end{abstract}

PALAVRAS-CHAVE: traumatismo raquimedular, reabilitação, psicologia.

\section{Coping strategies in rehabilitation of spinal cord injury}

ABSTRACT - International research centers are developing studies on spinal cord injury, aiming to improve rehabilitation-geared interventions and quality of life for patients. Considering the present scientific and professional interests of Health Psychology, this paper aims at comprehending the coping strategies adopted by patient and their families/caregivers during participation in a rehabilitation program. The sample included six patient/family dyads. Participants were assessed before entrance and at the end of the program, th rough of The Ways of Coping Checklist- Revised and a semi-stru ct u red interview. Data indicate changes in the applied coping modalities prior to rehabilitation (religiousness, positive thoughts and seeking social support) and after rehabilitation (problem-focused, positive thoughts and seeking social support).

KEY WORDS: spinal cord injury, rehabilitation, psychology.

Atualmente, em razão da recrudescência de episódios associados à violência urbana, como os acidentes de trânsito e as agressões por arma de fogo, um expressivo de cidadãos em todo o mundo enfrenta o sofrimento e as limitações provocadas pela lesão medular traumática. Assim, com o aumento da participação social desses indivíduos acometidos por traumatismo raquimedular, diversos centros hospitalares internacionais vêm realizando investigações destinadas à ampliação de conhecimentos teóricos e técnicos nesse campo da saúde. Nesse sentido, alguns trabalhos vêm investigando as re ações e conseqüências do traumatismo para o sujeito e seu meio social, incluindo as expectativas de progresso em um programa de reabilitação. Giannatta sio $^{1}$, por exemplo, alerta que a lesão medular pro- voca quebra na unidade psicofísica do indivíduo que anteriormente funcionava de maneira adequada e que as mudanças corporais e limitações da ação provocam reações psicológicas diversas como depressão, isolamento, impulsividade reacional, agre ssividade e apatia.

Nessa perspectiva, destacam-se os estudos voltados para ampliação da compreensão do processo de enfrentamento das doenças e incapacidades. E mbora diversos sistemas de categorização tenham sido propostos para a delimitação conceitual de enfrentamento, a definição mais abrangente e pertinente é de que se trata de uma resposta destinada a diminuir os encargos físico, emocional e psicológico relacionados aos eventos estressantes do ciclo de vida. A partir dessa definição, estratégias de en-

\footnotetext{
${ }^{1}$ Psicóloga da Rede SARAH de Hospitais. Mestre em Psicologia pela Universidade de Brasília, Brasília, DF, Brasil, (UnB); ${ }^{2}$ Doutora pela Université de Paris X-Nanterre, Professora Adjunta UnB. Pesquisadora do Conselho Nacional de Desenvolvimento Científico e Tecnológico, Coordenadora do Laboratório da Saúde e Desenvolvimento Humano do Instituto de Psicologia da UnB.
}

Recebido 13 Setembro 2004, recebido na forma final 3 Dezembro 2004. Aceito 3 Fevereiro 2005.

Dra. Maria Eloá Moreira da Silva Martins Pereira - SHIS QL 4 Conjunto 3 Casa 2 - 71610-235 Brasília DF - Brasil. E-mail: eloa@bsb.sarah.br ouaraujotc@unb.br 
frentamento são aquelas respostas que são efetivas em reduzir uma angústia imediata ou que contribuem para melhores resultados a longo prazo $^{2}$. Vale explicar que existem cinco principais abordag e $\mathrm{n} \mathrm{s}$ no estudo do enfrentamento. A primeira delas é baseada no sujeito, visto que assume constância a mbiental e considera ser a pessoa, o principal fator que afeta a mobilização das estratégias de enfrentamento. A abordagem baseada na situação dá destaque significativo às demandas ambientais na mobilização de estratégias, incluindo as características dos outros indivíduos. A terceira abordagem é de caráter interacionista, já que enfatiza pessoa e situação. A quarta abordagem é transacionista, pois defende a perspectiva de um processo envolvendo a pessoa, a situação e a modalidade de enfrentamento adotada. Para a quinta abordagem, o enfrentamento é resultante de avaliações tanto das situações quanto da experiência pessoal, o que definiria sua natureza cognitiva ${ }^{3}$.

No Brasil, a Rede SARAH é uma instituição dedicada à ortopedia e à reabilitação do grande incapacitado físico e ao tratamento de deformidades, traumas, doenças e infecções do aparelho locomotor. O Programa de Reabilitação do Lesado Medular é organizado por equipe multiprofissional, cuja principal meta consiste em viabilizar autonomia e qualidade de vida por meio de atividades voltadas para o desenvolvimento físico e sócio-educativo, envolvendo informação e orientação individual e em grupo para o paciente e seu familiar/cuidador.

Considerando, portanto, o interesse social e científico das questões associadas à reabilitação em casos de traumatismo raquimedular, a presente pesqui$\mathrm{sa}^{4}$ teve como objetivo geral investigar as estratégias de enfrentamento adotadas pelo paciente e seu acompanhante durante a participação em um programa de reabilitação hospitalar, visando: a) descrever e compreender as estratégias de enfrentamento desenvolvidas pelo paciente participante de um $\mathrm{p}$ rograma de reabilitação e seu familiar/acompanhante; b) identificar e avaliar diferenças nas estratégias de enfrentamento adotadas no momento de admissão (pré-reabilitação); e no momento de alta (pósreabilitação); e c) compreender a influência das atividades do programa de reabilitação no enfre ntamento do paciente e seu familiar/acompanhante.

\section{MÉTODO}

Sujeitos - Foram estudadas seis díades, constituídas por pacientes e seus familiares, acompanhadas pelo programa de reabilitação para indivíduos com lesão medular em hospital especializado em reabilitação no Distrito
Federal. Alguns critérios de exclusão foram definidos para o grupo de pacientes: lesão medular na infância, indivíduos com história psiquiátrica, analfabetismo ou escolaridade inferior a segunda série do ensino fundamental, co-morbidades, tentativa de suicídio, realização de tratamento exclusivamente cirúrgico, reabilitação em outra instituição e interrupção do programa. Então, constituíram a amostra dessa pesquisa: cinco sujeitos do sexo masculino e um do sexo feminino entre 19 e 42 anos de idade. Quanto à atividade anteriormente exercida, dois pacientes informaram que eram estudantes de curso superior, um estava desempregado, um era operador de máquinas, um trabalhava como agricultor e o outro estava empregado em uma serraria. Três eram solteiros, dois casados com filhos e um tinha relação marital estável. Cinco eram tetraplégicos e um paraplégico. A etiologia da lesão foi projétil de arma de fogo $(\mathrm{N}=1)$; queda de altura $(\mathrm{N}=1)$, me rgulho em águas rasas $(\mathrm{N}=1)$; queda de objeto (árvore) $(\mathrm{N}=1)$; queda em esporte $(\mathrm{N}=1)$ e acidente automobilístico $(\mathrm{N}=1) .0$ tempo decorrido desde a lesão foi de cinco meses a um ano $(\mathrm{N}=3)$, um ano a dois anos $(\mathrm{N}=2)$ e mais de dois anos $(\mathrm{N}=1)$. O período de internação foi de cinco semanas $(\mathrm{N}=5)$ e seis semanas $(\mathrm{N}=1)$. É importante explicar que esse período foi estipulado em razão de dois fatores. Primeiramente, um tempo mínimo de seis meses visa garantir que as conseqüências psicológicas imediatas à lesão (tais como, emoções conflitantes, desordem emocional e desorganização) não estejam exercendo forte influência ${ }^{5}$. Segundo, a escolha do período também foi n o rteada pela prática profissional, a qual indica ser esta uma fase de maior mobilização de recursos internos na busca de adaptação à situação da lesão.

O grupo de familiares foi composto por três mães, duas esposas e uma companheira entre 23 e 41 anos de idade. Três eram profissionais autônomas, uma estava dese m pregada e três eram "do lar". Três haviam concluído o ensino médio e três não concluíram o ensino fundamental. Os critérios de exclusão do referido grupo foram: história psiquiátrica, analfabetismo ou escolaridade inferior a segunda série do ensino fundamental.

Material - Foi utilizada a Escala Modos de Enfrentar P roblemas - The Ways of Coping Checklist - Revised, derivada do Modelo Transacional de Estresse de Lazarus ${ }^{6}$. Também foram aplicados quatro roteiros de entrevista semi-estruturada destinados respectivamente ao paciente em pré-reabilitação e em pós-reabilitação e ao familiar em pré-reabilitação e em pós-reabilitação. As entrevistas em pré-reabilitação incluíam questões pertinentes a: estágio do ciclo de vida do paciente, composição e org anização familiar, comunicação, rede de apoio, enfrentamento, rotinas e informações complementares (história da lesão medular, atendimentos médico-hospitalares e inte mações anteriores, história familiar anterior, situação vivenciada imediatamente anterior à lesão e escolha do acompanhante). Na etapa de pós-reabilitação, as entrevistas abarcavam questões relativas a: comunicação, rede 
de apoio, organização, enfrentamento, expectativa, rotina/perspectiva de futuro e atividades que os participantes avaliavam como as mais significativas no progra ma de reabilitação/ treino familiar.

Procedimentos - Na primeira semana de internação, previamente à realização de qualquer atividade específica de reabilitação, os sujeitos eram convidados a participar da pesquisa. Expressa sua aceitação voluntária, era apresentado um Termo de Consentimento Livre e Esclarecido (Resolução 196/96 do CNS/MS), o qual era lido pelo indivíduo e/ou pela pesquisadora. No caso dos pacientes com tetraplegia, com impedimento de movimentos para assinatura do documento, a autorização era gravada em áudio. Os instrumentos foram aplicados, individualmente, em uma única sessão, iniciando-se pela entrevista semiestruturada seguida da escala, em dois momentos distintos durante o período de internação: na semana de admissão e na semana de alta hospitalar. As díades paciente-familiar foram avaliadas no mesmo dia para evitar troca de informações. O tempo médio de realização da entrevista foi uma hora e dez minutos e a aplicação da escala exigiu aproximadamente 15 minutos. As entrevistas foram registradas em áudio após o consentimento do participante. As respostas relativas à escala foram marcadas pelo entrevistado ou pelo entrevistador, respeitando-se o desejo do participante e suas condições físicas. Caso a opção fosse pela auto-aplicação, o pesquisador se mantinha presente, junto ao sujeito, visando esclarecer possíveis dúvidas e orientando no que fosse necessário. As entrevistas foram integralmente transcritas para leitura e analisadas pelas duas pesquisadoras. Identificadas as unidades de significado, os respectivos trechos foram organizados em tópicos para posterior identificação de cada tema. Procedeu-se à análise das díades pacientefamiliar/acompanhante, de acordo com as fases de pré e pós-reabilitação, gerando um sistema de categorias temáticas. Além do depoimento dos participantes, a identificação das categorias relativas às modalidades de en- frentamento foi efetivada a partir das categorias apresentadas na Escala de Modos de Enfrentar Problemas: focalização no problema ("Encontro diferentes soluções para o meu problema"); religiosidade ("Pratico mais a religião desde que tenho esse problema"); pensamento fantasioso/desiderativo ("Eu sonho ou imagino um tempo ou lugar melhor do que aquele em que estou"); culpabilização dos outros ("Demonstro raiva para as pessoas ou coisas que causaram o problema"); auto-culpa ("Eu me sinto mal por não ter podido evitar o problema"); esquiva ("Durmo mais do que o habitual"); pensamento positivo ("Eu acho que estou em melhor situação do que outras pessoas"); busca de suporte social ("Converso com alguém que possa fazer alguma coisa para resolver o meu problema"). Foram obtidos os escores individuais finais e parciais em cada sub-escala, sendo que para a presente investigação estabeleceu-se como critério de estratégias mais utilizadas aquelas que apresentassem escores iguais ou superiores a três. Realizou-se, ainda, análise comparativa dos dados das entrevistas e da Escala.

\section{RESULTADOS}

a) Dados obtidos pela Escala Modos de Enfrentar Problemas de Vitaliano et al. No grupo de pacientes, as estratégias utilizadas com maior freqüência em pré-reabilitação e pós-reabilitação foram: religiosidade, pensamento desiderativo/fantasioso, pensamento positivo, focalização no problema e busca de suporte social. Não se evidenciou o uso da estratégia culpabilização dos outros. É importante considerar que ocorreram mudanças individuais quanto à sua utilização. Observou-se que os pacientes alteraram as estratégias de enfrentamento utilizadas entreos momentos de pré e pós-reabilitação, sendo que apenas $\mathrm{P}(02)$ e $\mathrm{P}(05)$ mantiveram as mesmas modalidades (Gráficos 1 e 2).

No grupo de familiares, é possível evidenciar que as estratégias utilizadas com maior freqüência em

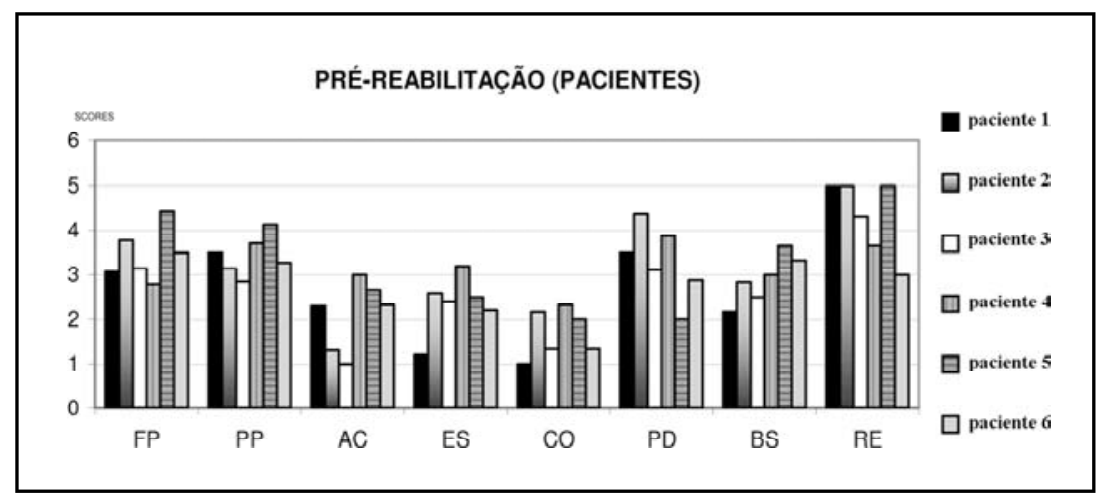

Gráfico 1. Estratégias de enfrentamento utilizadas por cada paciente em pré-rea bilitação. FP (focalização no problema), PP (pensamento positivo), AC (autoculpa), ES (esquiva), CO (culpabilização dos outros), PD (pensamento desiderativolfanta sioso), BS (busca de suporte social), RE (religiosidade). 


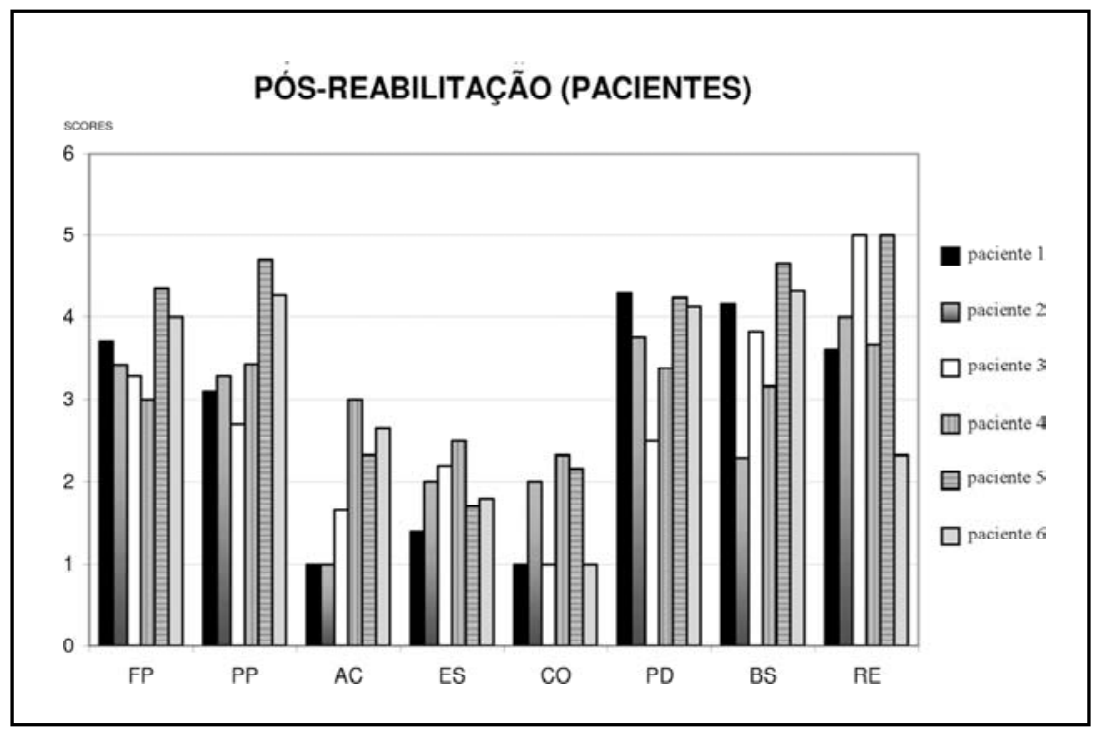

Gráfico 2. Estratégias de enfrentamento utilizadas por cada paciente em pós-reabi litação. FP, focalização no problema; $P P$, pensamento positivo; $A C$, autoculpa; $E S$, esquiva; $C O$, culpabilização dos outros; $P D$, pensamento desiderativo/fantasioso; $B S$, busca de suporte social; $R E$, religiosidade.

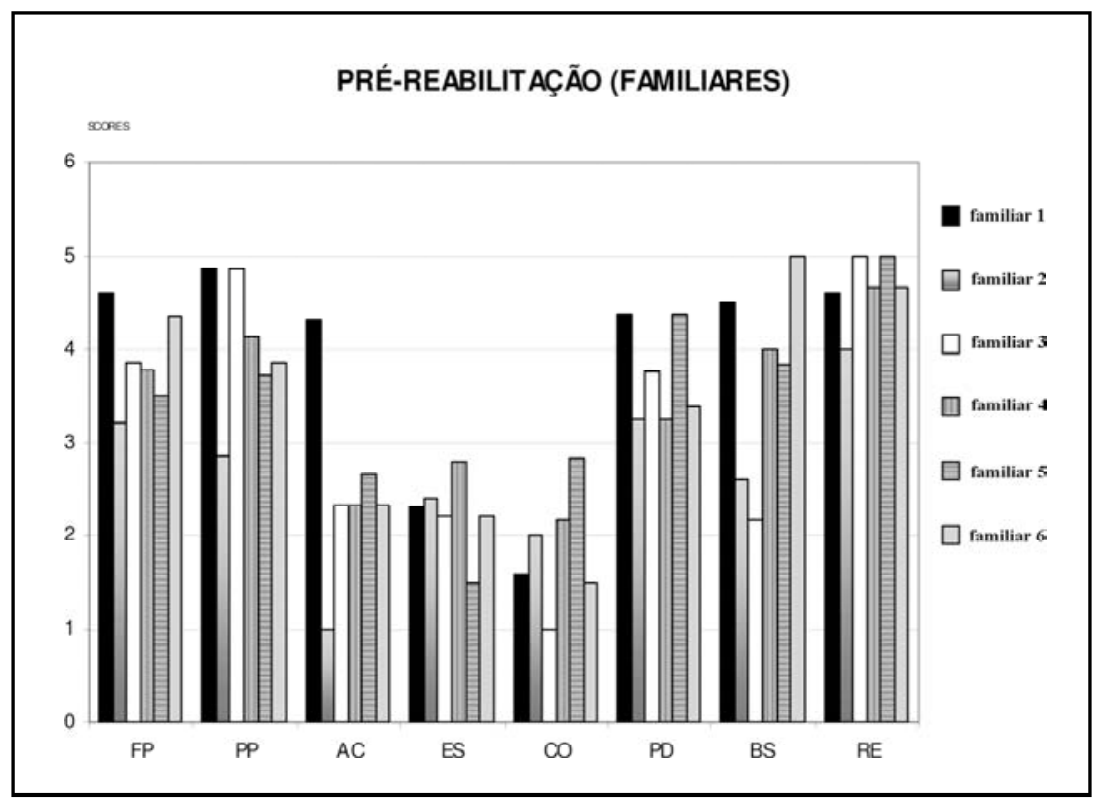

Gráfico 3. Estratégias de enfrentamento utilizadas por cada familiar em pré-reabili tação. FP, focalização no problema; PP, pensamento positivo; $A C$, autoculpa; $E S$, es quiva; $C O$, culpabilização dos outros; $P D$, pensamento desiderativo/fantasioso; $B S$, busca de suporte social; $R E$, religiosidade.

pré-reabilitação e pós-reabilitação foram: religiosidade, pensamento desiderativo/fantasioso, pensamento positivo, focalização no problema e busca de suporte social. Ao se comparar as duas etapas de pré e pós-reabilitação, verifica-se que $\mathrm{F}(04), \mathrm{F}(05)$ e $F(06)$ não apresentaram alterações quanto às modalidades de enfrentamento utilizadas. $F(01)$ acre scentou uma nova estratégia (esquiva), a qual era utilizada em menor freqüência quando comparada com as demais. Os participantes $\mathrm{F}(02)$ e $\mathrm{F}(03)$ acre scentaram uma estratégia (Gráficos 3 e 4).

Ao se analisar as díades paciente-acompanhante, observa-se que os familiares adotaram estratégias semelhantes aos dos pacientes, nas duas etapas. No entanto, é necessário considerar que, para quase todos os sujeitos, ocorreram mudanças individuais quanto a sua utilização. Também percebe-se que pacientes e familiares utilizaram estratégias idên- 


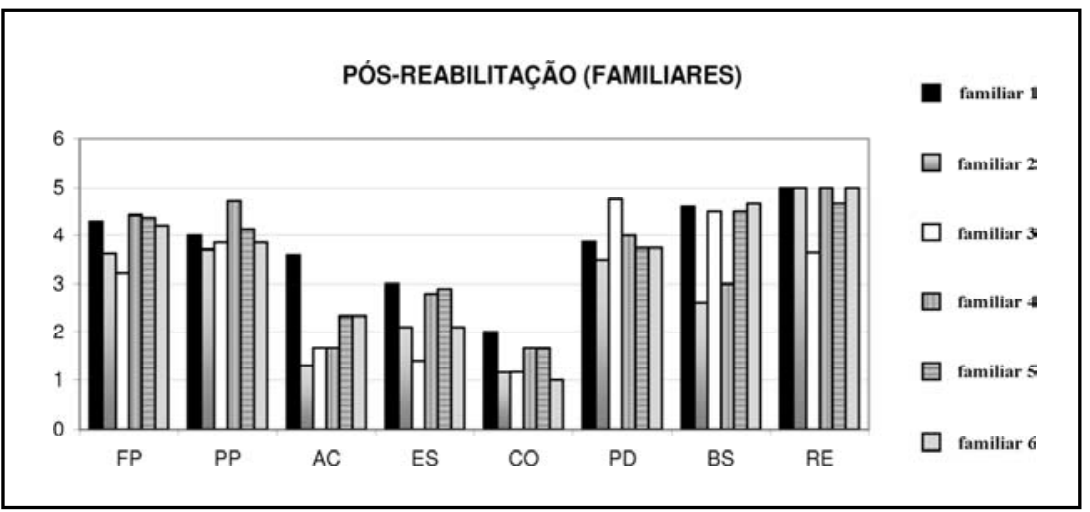

Gráfico 4. Estratégias de enfrentamento utilizadas por cada familiar em pós-reabilitação. FP, focalização no problema; PP, pensamento positivo; $A C$, autoculpa; ES, esquiva; $C O$, culpabilização dos outros; $P D$, pensamento desiderativo/fantasioso; $B S$, busca de suporte social; $R E$, religiosidade.

Tabela 1. Estratégias de enfrentamento nas etapas de pré e pós-reabilitação.

\begin{tabular}{|c|c|c|c|c|c|c|c|c|c|c|c|}
\hline \multicolumn{2}{|c|}{ Paciente 1} & \multicolumn{2}{|c|}{ Paciente 2} & \multicolumn{2}{|c|}{ Paciente 3} & \multicolumn{2}{|c|}{ Paciente 4} & \multicolumn{2}{|c|}{ Paciente 5} & \multicolumn{2}{|c|}{ Paciente 6} \\
\hline \multirow[t]{5}{*}{ Pré } & Pós & Pré & Pós & Pré & Pós & Pré & Pós & Pré & Pós & Pré & Pós \\
\hline & BS & & BS & & BS & BS & & BS & BS & BS & BS \\
\hline & FP & FP & FP & & FP & & FP & & FP & & FP \\
\hline & & & & & & $A C$ & & & & & \\
\hline & & & PP & PP & & & PP & & PP & PP & PP \\
\hline \multirow[t]{3}{*}{ Es } & & & & & & & & Es & & & \\
\hline & PD & PD & & PD & & & & & & & \\
\hline & & & & $\mathrm{Re}$ & & $\operatorname{Re}$ & $\operatorname{Re}$ & $\mathrm{Re}$ & & $\operatorname{Re}$ & \\
\hline $\mathrm{CO}$ & & & & & & & & & & & \\
\hline
\end{tabular}

FP, focalização no problema; PP, pensamento positivo; $A C$, autoculpa; ES, esquiva; CO, culpabilização dos outros; $P D$, pensamento desiderativo/fantasioso; $B S$, busca de suporte social; RE, religiosidade.

Tabela 2. Estratégias de enfrentamento pré e pós-reabilitação.

\begin{tabular}{|c|c|c|c|c|c|c|c|c|c|c|c|}
\hline \multicolumn{2}{|c|}{ Familiar 1} & \multicolumn{2}{|c|}{ Familiar 2} & \multicolumn{2}{|c|}{ Familiar 3} & \multicolumn{2}{|c|}{ Familiar 4} & \multicolumn{2}{|c|}{ Familiar 5} & \multicolumn{2}{|c|}{ Familiar 6} \\
\hline Pré & Pós & Pré & Pós & Pré & Pós & Pré & Pós & Pré & Pós & Pré & Pós \\
\hline BS & BS & & & & & BS & BS & BS & BS & BS & BS \\
\hline $\mathrm{FP}$ & $\mathrm{FP}$ & & FP & & FP & & & & $\mathrm{FP}$ & & \\
\hline \multirow[t]{6}{*}{$A C$} & & & & & & & $A C$ & & & & \\
\hline & & & PP & & PP & PP & PP & PP & PP & & PP \\
\hline & & Es & & Es & & & & & & & \\
\hline & & & PD & & & & & & & PD & \\
\hline & & & & $\mathrm{Re}$ & $\mathrm{Re}$ & $\operatorname{Re}$ & $\operatorname{Re}$ & & & $\operatorname{Re}$ & $\operatorname{Re}$ \\
\hline & & & & $\mathrm{CO}$ & & & & & & & \\
\hline
\end{tabular}

FP, focalização no problema; $P$, pensamento positivo; $A C$, autoculpa; ES, esquiva; $C O$, culpabilização dos outros; $P D$, pensamento desiderativo/fantasioso; $B S$, busca de suporte social; $R E$, religiosidade).

ticas somente no caso da díade $\mathrm{P}(05) / \mathrm{F}(05)$. Nos demais casos, evidencia-se discreta diferença, ocorre $n$ do manutenção de pelo menos três estratégias igualmente utilizadas nas fases de pré e pós-reabilitação. Em ambos os grupos, as mudanças ocorreram em relação à freqüência de utilização das estratégias, substituição ou acréscimo de novas estratégias.

b) Dados obtidos pelas entrevistas - Com relação ao grupo de pacientes, observa-se que ocorreram mudanças qualitativas quanto às modalidades de estratégias utilizadas, entre as etapas de pré e pósreabilitação, particularmente para $\mathrm{P}(01)$ e $\mathrm{P}(03)$, os quais apresentaram mudança daquelas anteriormente utilizadas. Os participantes $\mathrm{P}(02), \mathrm{P}(04)$ e $\mathrm{P}(05)$ a $p$ resentaram mudança de duas estratégias de um total de três utilizadas na etapa pré-reabilitação e $\mathrm{P}(06)$ apresentou mudança discreta, tendo somente substituído religiosidade por focalização no problema (Tabela 1).

Com relação ao grupo de familiares, comparan- 
do-se as estratégias utilizadas entre as etapas de pré e pós-reabilitação observam-se mudanças qualitativas (Tabela 2).

Ao se analisar as díades paciente-acompanhante, constata-se que na etapa de pré-reabilitação o correu utilização mais freqüente das estratégias: religiosidade, busca de suporte social, seguidas por pensamento positivo e esquiva. Na etapa de pósreabilitação, as estratégias mais utilizadas foram: busca de suporte social, focalização no problema e pensamento positivo. Vale destacar que religiosidade foi apresentada como a estratégia mais freqüentemente utilizada apenas pelo grupo dos familiares.

c) Estratégias de enfrentamento identificadas pela Escala e entrevistas - A partir dos dados apresentados pela análise dos dois instrumentos, evidenciou-se que as estratégias religiosidade, p e nsamento positivo e busca de suporte social foram as modalidades mais utilizadas pelos sujeitos na etapa de pré-reabilitação. Já no momento de pós-reabilitação, as estratégias mais freqüentemente apresentadas, por ambos os grupos, foram: focalização no problema, pensamento positivo e busca de suporte social.

\section{DISCUSSÃO}

Os dados apontam que na pré-reabilitação existe maior diferenciação entre as estratégias utilizadas por pacientes e familiares, enquanto que, na pósreabilitação, as modalidades adotadas tendem a se assemelhar. É possível, então, hipotetizar que o p rograma de reabilitação influencia o modo de enfrentamento dos participantes, podendo mobilizar os sujeitos a buscar estratégias que viabilizem uma melhor adaptação à situação da lesão medular. A clareza das informações e o repasse consistente das orientações possibilitarão uma reorganização da vida não somente do portador da lesão medular, mas também dos integrantes da rede sócio-familiar na qual ele se encontra, contribuindo, desta maneira, para a melhoria da qualidade de vida de todos os implicados. As evidências encontradas na presente investigação conduzem ao reconhecimento da relevância da atuação do profissional de psicologia enquanto membro da equipe de reabilitação, tendo em vista sua formação e treinamento como facilitador da comunicação individual e grupal. De acordo com os dados obtidos, o atendimento à díade deve ser privilegiado em intervenções psicológicas, visando à ampliação e aprimoramento da comunicação entre paciente e familiar/acompanhante. Todavia, as tarefas de facilitação da comunicação não são exclusivas do psicólogo do serviço, devendo ser sustentadas por todos os membros da equipe. Para tanto, é necessário assegurar treinamento contínuo em habilidades de comunicação para estes profissionais de saúde.

Em conclusão, foi possível observar mudanças quanto às modalidades de enfrentamento adotadas, ao se comparar as etapas de pré e de pós-reabilitação. A participação em atividades do programa de reabilitação, a percepção de aquisição pro g ressiva de independência, bem como a convivência com outros pacientes e familiares podem ser os fatores que promovem uma avaliação menos ameaçadora da situação de lesão, mobilizando os indivíduos no sentido de uma adaptação sem risco de acomodação. Após realizarem tais experiências, os participantes dessa pesquisa mostraram-se engajados em planos de alteração de rotina, visando alcançar melhoria da qualidade de vida. No caso específico do familiar, sua participação no programa de reabilitação dá oportunidade para o desenvolvimento do seu senso de competência, capacitandoo a conhecer o problema e a desempenhar seu papel de cuidador.

Sugerese que futuras investigações verifiquem a influência dos fatores encontrados nesse trabalho, no intuito de subsidiar novas diretrizes de condução do tratamento por parte da equipe de reabilitação. Recomenda-se, ainda, a execução de uma investigação prospectiva (por exemplo, seis meses após a alta hospitalar), visando identificar a estabilidade das modalidades de enfrentamento utilizadas por indivíduos afastados do ambiente hospitalar. Além do que, a realização de um estudo longitudinal, com maior amplitude temporal, contribuiria para uma melhor compreensão das estratégias de enfrentamento ao longo dos distintos estágios do ciclo de vida, tanto em relação ao paciente como ao seu familiar.

\section{REFERÊNCIAS}

1. Giannattasio ER. Aspectos psicológicos do lesado medular. Trabalho a p resentado no Curso: Reabilitação do Lesado Medular da AACD. São Paulo, 1988.

2. Snyder CR, Dinoff BL. Coping: where have you been? In Snyder CR (ed) Coping: the psychology of what works. New York: Oxford Univ Press, 1999.

3. Aldwin, CM. Stress, coping and development: an integrative perspective. London: Guilfors Press, 1994.

4. Pe reira MEMSM. Aspectos psicológicos da reabilitação em traumatismo raquimedular: modalidades de enfrentamento do paciente e seu familiar / acompanhante. Dissertação de Mestrado, Universidade de Brasília, Brasília, 2002.

5. North NT. The psychological effects of spinal cord injury: a review. Spinal Cord 1999;37:671-679.

6. Vitaliano PP, Russo J J, Carr JE, Maiuro RD, Becker J. The ways of coping checklist revision and psychometric properties. Mult Behav Res 1985;20:3-26. 